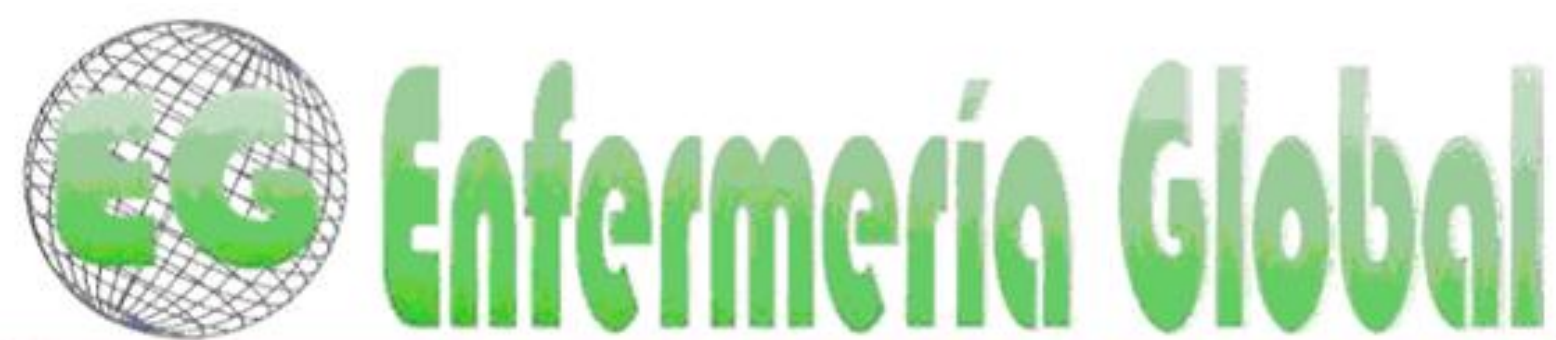

ISSN 1695-6141

Revista electrónica trimestral de Enfermería

$N^{\circ} 44$

Octubre 2016

www.um.es/egloball

REVISIONES

\title{
Análisis bibliométrico de los artículos sobre cuidado de enfermería publicados en revistas colombianas
}

Bibliometric analysis of articles on nursing care published in colombian magazines

\section{*Camacho Rodríguez, Doriam Esperanza ** Oviedo Córdoba, Haidy Rocío ${ }^{* * *}$ Ramos de la Hoz, Ediltrudis ****González Noguera, Tatiana Cecilia}

\author{
*Enfermera. Magister en Gestión de la calidad, seguridad y medio ambiente. Coordinadora de \\ Investigación. Universidad Cooperativa de Colombia Sede Bucaramanga. E-mail: \\ doricama@yahoo.es $\quad * *$ Magister en Enfermería. Decana Facultad de Enfermería. Universidad \\ Cooperativa de Colombia. Sede Santa Marta. ***Magister en Enfermería. Coordinadora Programa de \\ Enfermería. Universidad del Magdalena. ${ }^{* * * *}$ Magister en Enfermería. Profesora Facultad de Enfermería \\ Universidad del Magdalena. Colombia
}

Palabras clave: bibliometría, cuidado de enfermería, publicaciones, (Fuente: DeCS)

Keywords: bibliometrics; nursing care; publicactions (Source: MeSH).

\section{RESUMEN}

Objetivo: Realizar un análisis bibliométrico de los artículos sobre cuidado de enfermería, publicados en revistas Colombianas.

Materiales y método: Estudio bibliométrico descriptivo, retrospectivo. Se analizaron 95 artículos publicados en 18 revistas colombianas indexadas en Publindex, durante los años 2010 a 2015. La búsqueda se realizó mediante los descriptores: cuidado, modelos de enfermería, teoría de enfermería. El análisis descriptivo se realizó mediante una hoja de Excel y se analizaron los indicadores bibliométricos de productividad, citación, contenido y metodológicos.

Resultados: Se observa un incremento en la publicación en los últimos 5 años, siendo los artículos de investigaciones originales los que más se publican y las Instituciones que más publican son las Universidades de Bogotá y Antioquia. En relación con los indicadores de citación, el Índice de Price fue de $37,45 \%$, en tanto que el índice de aislamiento fue de 33,67\% y el índice de auto citación fue de $1,85 \%$. Sobre los indicadores de colaboración, el índice de coautoría fue de 2,7 y el índice de colaboración institucional fue de 1,3.

Conclusiones: Se observa un incremento en la producción científica de Enfermería en Colombia, no obstante, se hace necesario ampliar los indicadores de colaboración. 


\title{
ABSTRACT
}

Objective: To accomplished a bibliometric analysis of articles published on nursing care in Colombian journals.

\begin{abstract}
Materials and Methods: Descriptive y retrospective bibliometric study. It was analyzed 95 articles published in 18 Colombian indexed journals in Publindex during the years 2010 to 2015. The search was conducted by the care descriptors, nursing models, nursing theory. The descriptive analysis was performed using an Excel spreadsheet and bibliometric indicators of productivity, citation, content and methodology.
\end{abstract}

Results: An increase in publishing in the last 5 years is observed, being the original research articles the ones which are published the most and the institutions that published the most are Universities from Bogota and Antioquia. According to the citation indicators the index price was $37.45 \%$, while the isolation rate was $33.67 \%$ and self-citation index was $1.85 \%$. On indicators of collaboration, coauthorship index was 2.7 and the institutional collaboration index was 1.3.

Conclusions: An increase in scientific research about Nursing in Colombia is observed, however, it is necessary to expand cooperation indicators.

\section{INTRODUCCIÓN}

La investigación de Enfermería en Colombia se ha incrementado en los últimos años gracias a factores como la diversidad de programas de postgrado, la generación de sociedades científicas y el incremento de espacios de divulgación científica ${ }^{(1)}$. Dicha producción es necesaria para legitimar la Enfermería como profesión, mediante el desarrollo de un cuerpo de conocimientos necesario para la prestación de cuidados de salud a la población ${ }^{(2)}$, no obstante, no todos los trabajos de investigación se publican porque el ejercicio de publicación de resultados de investigación está ligado a los programas de posgrado ${ }^{(3)}$. Dada la importancia de la racionalización de la práctica de enfermería y la adición de nuevos conocimientos a la atención basada en la evidencia, es imprescindible para la profesión poder difundir los resultados de la investigación ${ }^{(4)}$, recurriendo a la publicación en revistas indexadas y la búsqueda de alianzas estratégicas para fortalecer los grupos de investigación ${ }^{(5)}$.

Una de las estrategias para determinar el avance en la investigación en una determinada área es la realización de estudios bibliométricos; al respecto, se encontraron diversos artículos donde se analizan publicaciones sobre temas específicos como geriatría ${ }^{(6)}$, urgencias cardiacas y coronarias ${ }^{(7)}$, cuidados intensivos y coronarios ${ }^{(8)}$, así como la producción de revistas de Enfermería en forma individual, como la Revista de Enfermería en cardiología ${ }^{(9)}$, Cuiden ${ }^{(10)}$, Rol de Enfermería ${ }^{(11)}$ publicaciones en bases de datos de nivel internacional, como ISI Web of Science (12) pero no específicamente de las Revistas Colombianas. La Enfermería en Colombia ha producido investigaciones en diversos temas, no obstante, el interés del presente trabajo es indagar acerca de la producción en el tema específico de cuidado de Enfermería. Para ello se recurre al análisis de indicadores bibliométricos, los cuales permiten evaluar, determinar y proporcionar información sobre los resultados del crecimiento en el proceso investigativo en cualquier campo de la ciencia ${ }^{(13)}$.

Para el análisis bibliométrico se puede utilizar la clasificación de Spinak (1998) quien los clasifica en dos grandes grupos, los indicadores de actividad y los de impacto. Los indicadores de actividad representan el estado real de la ciencia e incluyen número y distribución de publicaciones, productividad, dispersión de las publicaciones, colaboración en las publicaciones, vida media de la citación o envejecimiento, 
conexiones entre autores, entre otros, mientras que entre los indicadores de impacto se encuentran la evaluación de documentos muy citados "Hot papers" y el factor de impacto ${ }^{(14)}$, empleado por la publicación del Science Citation Index -SCl- ${ }^{(15)}$. Otra clasificación es la de Cano y Bueno (1999) que divide los indicadores en personales, de productividad (índices de colaboración, multiautoría e institucionalidad), citación (factor de impacto de las revistas e índices de obsolescencia, inmediatez, de actualidad temática, aislamiento y autocitación), indicadores de contenido (temáticos o contextuales, descriptores) e índices metodológicos (teoría desde o para la que se trabaja y diseños específicos utilizados) $\left({ }^{16}\right)$. Algunos de los indicadores bibliométricos más utilizados son los dos obsolescencia de Burton y Kebler, el Índice de Price o porcentaje de referencias con menos de 5 años de antigüedad y el índice de aislamiento (insularity) o porcentaje de referencias que corresponden a publicaciones del mismo país de la publicación ${ }^{(17)}$.

\section{MATERIALES Y MÉTODO}

Se realizó un estudio bibliométrico descriptivo, retrospectivo, de la producción científica de revistas Colombianas sobre cuidado de enfermería publicada de 2005 a 2015. Se analizaron los artículos publicados en 18 revistas colombianas indexadas en Publindex, 9 de enfermería y 9 del área de la salud, durante los años 2010 a 2015, a través de la consulta en las páginas web de cada revista. La búsqueda se realizó mediante los descriptores cuidado, modelos de enfermería, teoría de enfermería, encontrándose 95 artículos que cumplían con el requisito de la presencia del descriptor en el título del mismo.

Los datos fueron analizados en una hoja de cálculo de Excel con diferentes variables a analizar, entre ellas la revista, el tema, Institución de filiación de los autores, tipo de artículo, año de publicación, número de autores, sujetos de estudio y abordaje del estudio. Finalmente se utilizó la estadística descriptiva para analizar los resultados. Los indicadores bibliométricos analziados fueron los de productividad, citación, contenido y metodológicos, según la clasificación de Cano y Bueno ${ }^{(16)}$.

\section{RESULTADOS}

Se analizaron 95 artículos publicados en 18 revistas indexadas de Colombia, de los cuales el $22 \%(n=21)$ son de origen extranjero, provenientes de Brasil $(n=7 ; 7,4 \%)$, México $(n=7 ; 7,4 \%)$, Chile $(n=4 ; 4,2 \%)$ y España $(n=3 ; 3,2 \%)$. La Región de Colombia donde más se encontraron artículos sobre el tema fue Bogotá, debido a que allí se encuentran 6 de las 18 revistas analizadas, siendo mayor la producción en las Revistas Aquichán ( $\mathrm{n}=28 ; 27 \%)$, Investigación en Enfermería, Imagen y desarrollo $(n=13 ; 12 \%)$ y Avances en Enfermería $(n=11 ; 10 \%)$. Por otra parte, la mayoría de artículos se encontraron en las Revistas específicas de Enfermería ( $n=88 ; 84 \%)$, en tanto que el porcentaje restante se publicaron en Revistas del área de la salud (Figura 1). 
Figura 1. Distribución de las Revistas Colombianas según el volumen de artículos publicados sobre el tema

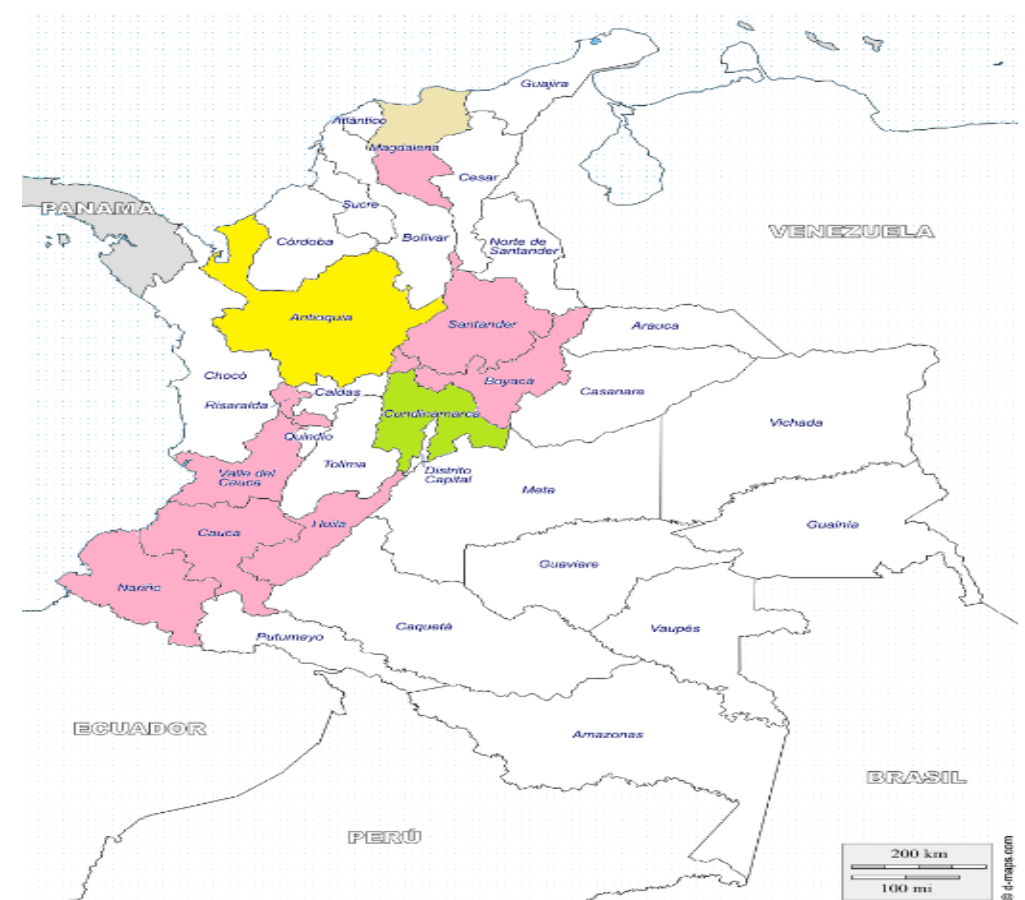

Convenciones de los colores: rosado $=0$ a 9 artículos, amarillo $=10$ a 18 y verde $=19$ a 28 .

(Elaborado en base al mapa de Colombia publicado en http://www.dmaps.com/carte.php?\&num_car=4094\&lang=es)

En Colombia, la publicación de artículos sobre el tema específico de cuidado de enfermería se ha incrementado con los años, siendo mayor la producción de artículos originales ( $n=65 ; 68,4 \%$ ), seguido por los de revisión $(n=26 ; 27,4 \%)$ y los reportes de caso $(n=4 ; 4,2 \%)$ (Figura 2$)$.

Figura 2. Distribución por años y tipología de los artículos publicados

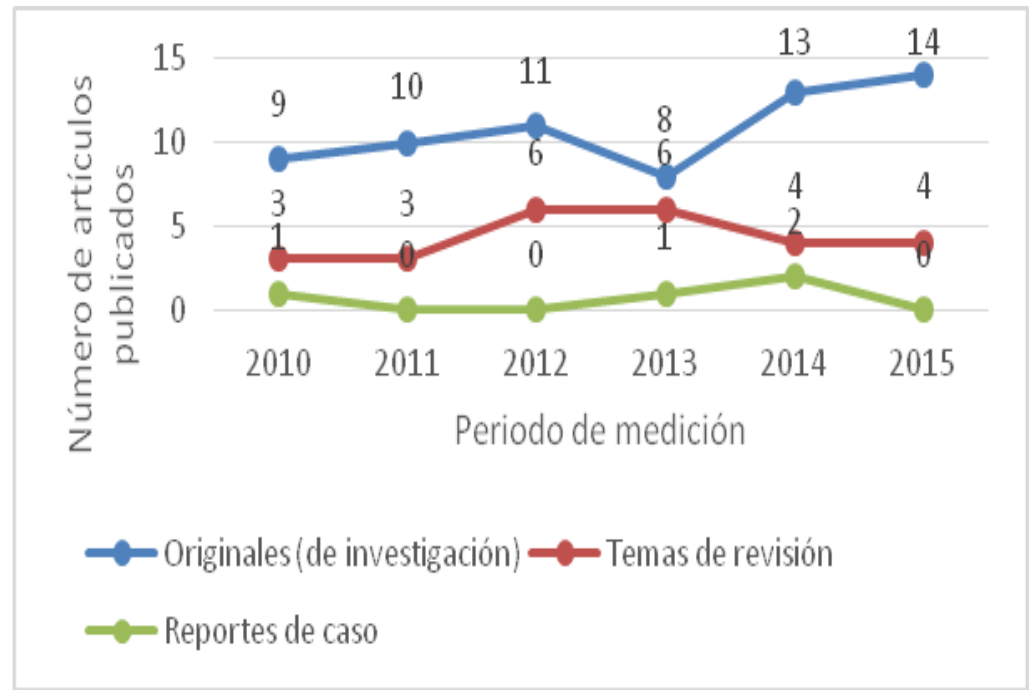


Indicadores de colaboración: Para los 95 artículos analizados se encontraron 258 autores. El índice de coautoría o número de firmas por trabajo fue de 2,7, el índice de colaboración institucional fue de 1,3. Al respecto, las Instituciones con mayor número de publicaciones, según la filiación del primer autor son en su orden: Universidad Nacional $(n=15 ; 15,8 \%)$, Universidad Javeriana $(n=8 ; 8,4 \%)$, Universidad de Antioquia $(n=6 ; 6,3 \%)$ y Universidad del Bosque $(n=5 ; 5,3 \%)$. Cabe destacar que también se encontraron publicaciones de Instituciones de salud como el Hospital Universitario Fundación Santa Fe de Bogotá $(n=3 ; 3,2 \%)$ y el Hospital Hernando Moncaleano de Neiva $(n=1 ; 1,1 \%)$. En relación con las Instituciones extranjeras que han publicado en Colombia sobre el tema de cuidado, predomina la Universidad Federal do Rio Grande do Soul, Universidad de Rio de Janeiro, Universidad Autónoma de Nuevo León y Universidad de Concepción de Chile, cada una con dos artículos publicados respectivamente.

Indicadores de citación: El Índice de obsolescencia o de envejecimiento se midió a través del índice de Price, el cual fue de 37,45\%, el índice de aislamiento fue de $33,67 \%$ y el índice de auto citación fue de 1,85\%. De igual forma se analizó la mediana de las referencias, la cual fue de $26,7+/-3$.

Índice de impacto: Se revisó el índice de impacto de las Revistas consultadas según SCImago Journal \& Country Rank, para las revistas indexadas en Scopus, así como la categoría de las Revistas según Plublindex (Ver tabla I.).

Tabla I. Índice de impactos e Indexación de las Revistas consultadas

\begin{tabular}{|c|c|c|c|c|}
\hline Revista & Institución editora & $\begin{array}{l}\text { Categoría } \\
\text { Publindex }\end{array}$ & $\begin{array}{l}\text { Quartil } \\
\text { (SJR- } \\
\text { SCOPUS) }\end{array}$ & Impacto \\
\hline Aquichan & Universidad de la Sabana & A1 & 3 & 0,15 \\
\hline Colombia médica & Universidad del Valle & A1 & - & - \\
\hline $\begin{array}{ll}\text { Avances } & \text { en } \\
\text { enfermería } & \\
\end{array}$ & $\begin{array}{lll}\text { Universidad Nacional de } \\
\text { Colombia }\end{array}$ & A2 & - & - \\
\hline $\begin{array}{l}\text { Hacia la promoción } \\
\text { de la salud }\end{array}$ & Universidad de Caldas & A2 & - & - \\
\hline $\begin{array}{lr}\text { Investigación } & \text { y } \\
\text { educación } & \text { en } \\
\text { enfermería } & \end{array}$ & Universidad de Antioquia & A2 & - & - \\
\hline $\begin{array}{l}\text { Revista ciencias de } \\
\text { la salud }\end{array}$ & $\begin{array}{l}\text { Colegio Mayor de Nuestra } \\
\text { Señora del Rosario }\end{array}$ & A2 & 4 & 0,1 \\
\hline Universidad y salud & Universidad de Nariño & $\mathrm{A} 2$ & & \\
\hline $\begin{array}{l}\text { Investigación en } \\
\text { enfermería: imagen } \\
\text { y desarrollo }\end{array}$ & $\begin{array}{l}\text { Pontificia Universidad } \\
\text { Javeriana - Sede Bogotá }\end{array}$ & $\mathrm{B}$ & - & - \\
\hline Medicas UIS & $\begin{array}{l}\text { Universidad Industrial de } \\
\text { Santander }\end{array}$ & $B$ & - & - \\
\hline $\begin{array}{l}\text { Actualizaciones en } \\
\text { enfermería }\end{array}$ & $\begin{array}{l}\text { Fundación Santa Fé de } \\
\text { Bogotá }\end{array}$ & $\mathrm{C}$ & - & - \\
\hline Duazary & Universidad del Magdalena & $\mathrm{C}$ & - & - \\
\hline $\begin{array}{l}\text { Revista Facultad de } \\
\text { salud Universidad } \\
\text { Surcolombiana }\end{array}$ & Universidad Surcolombiana & $\mathrm{C}$ & - & - \\
\hline
\end{tabular}




\begin{tabular}{llccc} 
Revista & Institución editora & $\begin{array}{l}\text { Categoría } \\
\text { Publindex }\end{array}$ & $\begin{array}{c}\text { Quartil } \\
\text { (SJR- } \\
\text { SCOPUS) }\end{array}$ & Impacto \\
\hline $\begin{array}{l}\text { Cultura del cuidado } \\
\text { enfermería }\end{array}$ & $\begin{array}{l}\text { Universidad Libre de } \\
\text { Colombia - Sede Pereira }\end{array}$ & C & - & - \\
\hline $\begin{array}{l}\text { Revista colombiana } \\
\text { salud libre }\end{array}$ & $\begin{array}{l}\text { Universidad Libre de } \\
\text { Colombia - Cali }\end{array}$ & C & - & - \\
\hline $\begin{array}{l}\text { Revista Colombiana } \\
\text { de Enfermería }\end{array}$ & Universidad El Bosque & C & - & - \\
\hline
\end{tabular}

Indicadores de contenido:

Índice temático o contextual: Los temas sobre los que más se realizan investigaciones son cuidado en situación de cronicidad, aplicación de la teoría en la práctica y Gestión del cuidado (Ver tabla II).

Tabla II. Temáticas de investigación y metodología utilizada

\begin{tabular}{|c|c|c|c|c|c|c|c|c|}
\hline \multirow[t]{2}{*}{$\begin{array}{l}\text { Temáticas } \\
\text { investigación }\end{array}$} & \multicolumn{2}{|c|}{$\begin{array}{l}\text { Metodología } \\
\text { cuantitativa }\end{array}$} & \multicolumn{2}{|c|}{$\begin{array}{l}\text { Metodología } \\
\text { cualitativa }\end{array}$} & \multicolumn{2}{|c|}{$\begin{array}{l}\text { Metodología } \\
\text { mixta }\end{array}$} & \multicolumn{2}{|c|}{ Total } \\
\hline & No. & $\%$ & No. & $\%$ & No. & $\%$ & No. & $\%$ \\
\hline $\begin{array}{l}\text { Cuidado de la madre y } \\
\text { el niño }\end{array}$ & 5 & 5,3 & 4 & 4,2 & - & - & 9 & 9,5 \\
\hline $\begin{array}{l}\text { Salud sexual } \quad \text { y } \\
\text { reproductiva }\end{array}$ & - & - & 2 & 2,1 & - & - & 9 & 9,5 \\
\hline $\begin{array}{lll}\text { Cuidado en } & \text { peri- } \\
\text { operatorio } & & \end{array}$ & 3 & 3,2 & 2 & 2,1 & 1 & 1,1 & 6 & 6,3 \\
\hline Gestión del cuidado & 11 & 11,6 & 1 & 1,1 & 2 & 2,1 & 14 & 14,7 \\
\hline Cuidado oncológico & 1 & 1,1 & 2 & 2,1 & - & - & 3 & 3,2 \\
\hline $\begin{array}{lr}\text { Educación } & \text { y } \\
\text { comunicación } & \text { en } \\
\text { Enfermería } & \end{array}$ & 4 & 4,2 & 2 & 2,1 & - & - & 6 & 6,3 \\
\hline $\begin{array}{ll}\text { Validación } & \text { de } \\
\text { instrumentos } & \end{array}$ & 6 & 6,3 & - & - & - & - & 6 & 6,3 \\
\hline $\begin{array}{l}\text { Cuidado en situación } \\
\text { de cronicidad }\end{array}$ & 10 & 10,5 & 9 & 9,5 & - & - & 19 & 20,0 \\
\hline $\begin{array}{l}\text { Aplicación de teoría en } \\
\text { la práctica }\end{array}$ & 11 & 11,6 & 4 & 4,2 & 1 & 1,1 & 16 & 16,8 \\
\hline Cuidado transcultural & 1 & 1,1 & 6 & 6,3 & - & - & 7 & 7,4 \\
\hline Salud mental & 2 & 2,1 & - & - & - & - & 2 & 2,1 \\
\hline Cuidado crítico & 4 & 4,2 & - & - & - & - & 4 & 4,2 \\
\hline Salud laboral & - & - & 1 & 1,1 & - & - & 1 & 1,1 \\
\hline Total & 58 & 61,1 & 33 & 34,7 & 4 & 4,2 & - & - \\
\hline
\end{tabular}

Descriptores: los descriptores más utilizados fueron enfermería (58), teoría de enfermería (11), cuidadores (10) y cuidado de enfermería (8).

Indicadores metodológicos:

Teoría desde o para la que se trabaja: Al analizar las teorías o modelos de Enfermería que se refieren en los trabajos de investigación se encontró que se aplicaron en su 
orden Dorothea Orem, Jean Watson, teoría de síntomas desagradables, Callista Roy, Imogene King y Modelo de cuidado de Kalish.

Figura 3. Teorías utilizadas en los artículos sobre cuidado de enfermería

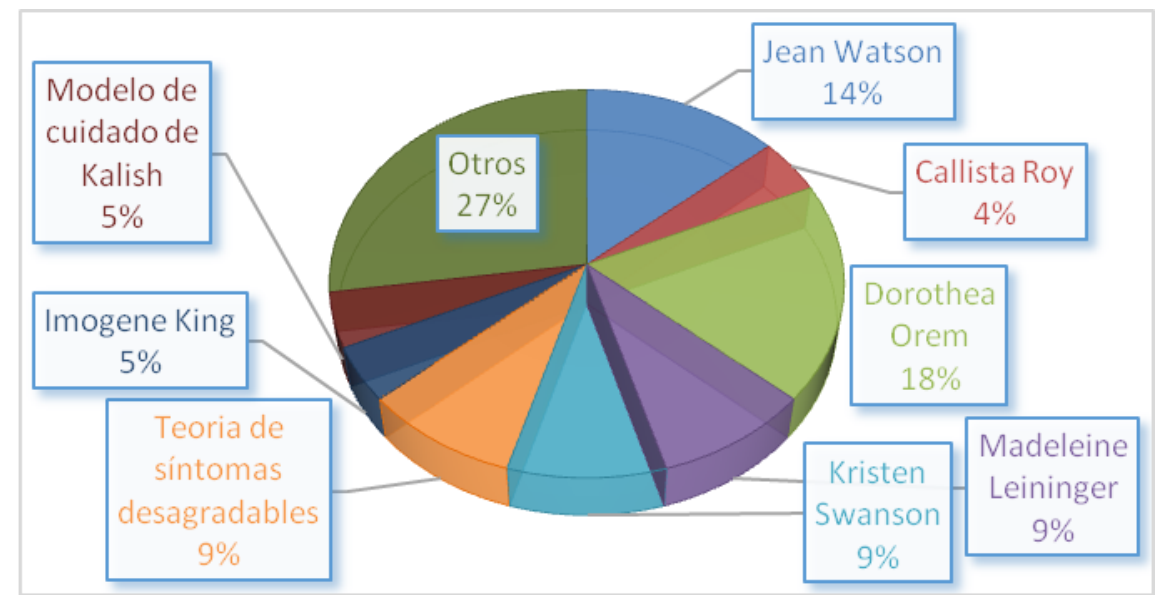

Diseños específicos utilizados: La mayoría de estudios fueron realizados con abordaje cuantitativo ( $n=58 ; 61,1 \%$ ), seguido por los estudios cualitativos ( $n=33 ; 34,7 \%)$ y los de metodología mixta $(n=4 ; 4,2 \%)$.

\section{DISCUSIÓN}

Las publicaciones sobre el tema de cuidado de Enfermería en Colombia se concentran en las Revistas de mayor impacto, tales como Aquichán, Investigación en Enfermería, Imagen y desarrollo y Avances en Enfermería; de hecho, en otro estudio realizado en Colombia se encontró que las Universidades que más publican son aquellas donde se concentra el $62.4 \%$ de la producción científica y condicionan además que sus autores estén dentro de los más productivos, entre las que sobresalen la Universidad Nacional de Colombia, Universidad de Antioquía, Universidad del Valle, Universidad de la Sabana y Pontificia Universidad Javeriana ${ }^{(12)}$. Al respecto, Johnstone afirma que si los autores de enfermería abandonan su compromiso editorial y publican sólo en las revistas "elite" (muchos de los cuales pueden ser de otros países), la capacidad de la profesión de enfermería para desarrollar y controlar la vanguardia de su conocimiento disciplinar se podría colocar en riesgo ${ }^{(18)}$.

Tradicionalmente las enfermeras del área de docencia son quienes más publican en Colombia ${ }^{(19)}$, de hecho en este estudio el $90 \%$ de los artículos fueron publicados por autores vinculados a las Universidades, porcentaje mayor al encontrado en otro estudio realizado en Colombia sobre publicaciones en bases de datos, donde el $59 \%$ de la producción se concentró en Universidades, aunque otras instituciones mantuvieron una importante presencia con sus aportes a la producción científica ${ }^{(12)}$. Por su parte, Alarcón y Astudillo encontraron que el $88 \%$ de los autores eran del ámbito universitario ${ }^{(1)}$.

La publicación de artículos originales es un indicador de que se ha incrementado la investigación en Colombia, lo que se evidencia en el alto porcentaje de artículos originales o de investigación en este estudio, similar a lo encontrado en otro estudio realizado en Colombia ${ }^{(12)}$. 
Al analizar los índices de colaboración se encontró que el índice de coautoría fue de 2,7 , bajo si se compara con el encontrado en el estudio de Chaviano ${ }^{(12)}$ donde el índice es de 6, lo cual representa una mayor colaboración entre los autores y que seguramente se traduce en una mayor riqueza en el contenido de la publicación (20).

Respecto del índice de impacto de las revistas, se debe anotar que solamente dos están indixadas en Scopus y por tanto tienen índice de impacto, siendo bajo si se tiene en cuenta que las revistas están en cuartiles 3 y 4 . Al respecto, se debe anotar que existen numerosas voces a favor y en contra de la medición del índice de impacto, ya que las condiciones de las revistas varían de país a país, e incluso de profesión a profesión. Es por ello que en Estados Unidos se construyeron índices de impacto para la evaluación de las publicaciones del área de enfermería y partería ${ }^{(21)}$.

Los temas que se trataron con mayor frecuencia en las investigaciones que dieron origen a los artículos fueron cuidado en situación de cronicidad, aplicación de la teoría en la práctica y Gestión del cuidado, este último tema coincide con los hallazgos de Fernández ${ }^{(22)}$ y Vélez quien además anota que dentro de las líneas temáticas que han sido abordadas de manera prioritaria en la investigación en enfermería en Colombia se encuentran epidemiología-salud colectiva, epistemología, gestión y gerencia $^{(5)}$.

Respecto del abordaje de la investigación, se encontró que prima el cuantitativo, similar a los resultados de un estudio realizado en Chile, donde el $80,8 \%$ utilizó el método cuantitativo, 17,3\% fueron estudios cualitativos, y sólo el 1,3\% utilizó un acercamiento mixto, cuali y cuantitativo ${ }^{(1)}$.

\section{CONCLUSIÓN}

Se observa un incremento en la producción científica de Enfermería en Colombia, predominando la publicación de artículos originales y particularmente sobre un tema que es importante para el desarrollo de Enfermería como disciplina profesional, como lo es la aplicación de la teoría en la práctica; no obstante, se hace necesario incrementar el índice de coautoría, así como el índice de colaboración Institucional. Cabe anotar que en Colombia no existen publicaciones especializadas, como ocurre en España, donde se encuentran publicaciones específicas para nefrología, historia de enfermería o matronas ${ }^{(6)}$.

Este estudio presenta una visión acerca de la producción sobre el tema de cuidado de Enfermería, no obstante, se hace necesario fomentar la realización de estudios bibliométricos sobre otros temas específicos de Enfermería, teniendo en cuenta que la productividad y la calidad de la investigación producida por investigadores individuales, grupos de investigación y las universidades son una medida importante de su éxito y su contribución a la productividad de la economía ${ }^{(23)}$.

\section{REFERENCIAS BIBLIOGRÁFICAS}

1. Alarcon, A., Astudillo P. La investigación en Enfermería en Revistas Latinoamericanas. Cienc y enfermería. Universidad de Concepción. 2007;13(2):25-31.

2. Vélez Vélez E. Investigación en Enfermería, fundamento de la disciplina. Rev Adm Sanit Siglo XXI.2009;07(02):341-56. 
3. Zavala MOQ, Klijn TP. Internacionalización de la Educación en Enfermería y sus Desafíos. Enferm. glob [online] 2011; 10(24). Disponible en http://scielo.isciii.es/pdf/eg/v10n24/reflexiones1.pdf

4. Wilkes L, Jackson D. Trends in publication of research papers by Australianbased nurse authors. Collegian. 2011;18(3):125-30.

5. Vélez Álvarez C, Pico Merchán ME, Ariza Olarte C, Castellanos F, Salazar Maya ÁM. Tendencias y agenda de prioridades de investigación en unidades académicas de Educación superior de Enfermería en Colombia. Cienc y enfermería. 2014;20(3):11-20.

6. Jiménez Navascués M. L, Hijar Ordovás CA. Estudio bibliométrico (2001-2009) sobre la enfermería geriátrica y gerontológica en España. Gerokomos. 2012;23(2):55-8.

7. Castro Teijeiro J. Producción científica en enfermería de urgencias cardíacas y coronarias: Análisis de la literatura. Enfermería Glob. 2010; (20). Disponible en http://scielo.isciii.es/pdf/eg/n20/revision1.pdf

8. Lizarbe Chocarro M. Enfermería en Cuidados Intensivos y Coronarios. Análisis bibliométrico de 180 artículos originales. Enfermería Intensiva. 2007;18(3):12637.

9. Medina García J, Martínez Casas J. Análisis de la producción científica de la revista Enfermería en cardiología en sus 20 años de edición. Enfermería en Cardiol. 2015;22(64):93-100.

10. García JM, Casas JMM, Leal $L$ marcela R. La comunicación científica de la investigación cualitativa en la Enfermería lberoamericana incluida en CUIDEN, en el año 2012. INDEX. 2014;8(20). Disponible en http://www.indexf.com/para/n20/pdf/196.pdf

11. Flores Martín J. Análisis bibliométrico de la revista Rol de Enfermería. Comparativo entre 2000 y 2004. Doc las Ciencias la Inf. 2004;31:17-43.

12. Gregorio-Chaviano O, Méndez-Rátiva CP, González MJP, Guzmán MF. Investigación colombiana en enfermería. Un análisis bibliométrico de su visibilidad en ISI WoS (2001-2013). Enferm Global. 2015; 14 (4): 175-91.

13. Escorcia-Otálora T. El análisis bibliométrico como herramienta para el seguimiento de publicaciones científicas, tesis y trabajos de grado. Pontificia Universidad Javeriana; 2008. Disponible en http://www.javeriana.edu.co/biblos/tesis/ciencias/tesis209.pdf

14. Spinak E. Indicadores cienciometricos. Ci Inf, Brasília. 1998;27(2):141-8.

15. Camps D. Limitaciones de los indicadores bibliométricos en la evaluación de la actividad científica biomédica. Colomb Med. 2008; 39(1):74-9.

16. Vallejo-Ruiz M. Estudio longitudinal de la producción Española de tesis doctorales en educación matemática (1975-2002). Universidad de Granada; 2005. Disponible en http://hera.ugr.es/tesisugr/15389807.pdf

17. Escorcia-Otálora, T., Poutou-Piñales R. Análisis bibliométrico de los artículos originales publicados en la revista Universitas Scientiarum (1987-2007).Univ Sci. 2008;13(3):236-44.

18. Johnstone M-J. Journal impact factors: implications for the nursing profession. Int Nurs Rev. 2007;54(1):35-40.

19. Melgarejo LM, Huertas SG. Caracterización de las publicaciones periódicas de la enfermería en Colombia visibles en Internet. Avances en Enfermería. 2011; 29(1):159-68.

20. Camps D. Estudio bibliométrico general de colaboración y consumo de la información en artículos originales de la revista Universitas Médica, período 2002 a 2006. Univ Médica. 2007;48(4):358-65. 
21. Crookes PA, Reis SL, Jones SC. The development of a ranking tool for refereed journals in which nursing and midwifery researchers publish their work. Nurse Educ Today. 2010;30(5):420-7.

22. Fernández Camiñas, J. y Regueiro Cabana M. Análisis de la producción científica de la enfermería en España en el año 2012. Cuad atención primaria. 2014;20:78-81.

23. Davidson, P., Newton, P., Ferguson,C., Daly, J., Elliott, D., Homer, C., Duffield, C \& Jackson D. Rating and Ranking the Role of Bibliometrics and Webometrics in Nursing and Midwifery. Sci World J. 2014;1-6.

Recibido: 23 de enero 2016; Acepado: 26 de marzo 2016 